Conclusion Clinically significant BMI changes, similar to those achieved under research conditions, may be replicable in service delivery settings for children of all socio-demographic groups analysed. However, at the population level, scaled up programmes may work better for some groups than others. Public health implications of these results for health inequalities will be discussed.

\section{OP06 WELL LONDON: RESULTS OF A CLUSTER-RANDOMISED TRIAL OF A COMMUNITY DEVELOPMENT APPROACH TO IMPROVING HEALTH BEHAVIOURS AND MENTAL WELLBEING IN DEPRIVED INNER-CITY NEIGHBOURHOODS}

doi:10.1136/jech-2012-201753.006

${ }^{1} \mathrm{G}$ Phillips, ${ }^{2} \mathrm{R}$ Hayes, ${ }^{2} \mathrm{C}$ Bottomley, ${ }^{3} \mathrm{M}$ Petticrew, ${ }^{1} \mathrm{P}$ Watts, ${ }^{3 \mathrm{~K}}$ Lock, ${ }^{4} \mathrm{~A}$ Clow, ${ }^{5} \mathrm{~A}$ Draper, ${ }^{6} \mathrm{D}$ Moore, ${ }^{7} \mathrm{E}$ Schmidt, ${ }^{1} \mathrm{P}$ Tobi, ${ }^{1} \mathrm{~S}$ Lais, ${ }^{1} \mathrm{G}$ Yu, ' $\mathrm{G}$ Barrow-Guevara, ${ }^{1} \mathrm{~A}$ Renton. ${ }^{\prime} / n s t i t u t e$ for Health and Human Development, University of East London, London, UK; ${ }^{2}$ Faculty of Epidemiology and Population Health, London School of Hygiene and Tropical Medicine (LSHTM), London, UK; ${ }^{3}$ Faculty of Public Health and Policy, LSHTM, London, UK; ${ }^{4} \mathrm{De}-$ partment of Psychology, University of Westminster, London, UK; ${ }^{5}$ Department of Human and Health Sciences, University of Westminster, London, UK; ${ }^{\circ} / n s t i t u t e$ for Research on Child Development, University of East London, London, UK

Background Few public health interventions combining modification of the social and built environment with individual-level health promotion have been robustly evaluated in the UK. Well London is an assets-based community development programme designed to improve physical activity, healthy eating and mental wellbeing in highly deprived inner-city communities. The programme, delivered between 2007 and 2011, comprised a mix of projects delivering traditional health promotion, community development and changes to the physical neighbourhood environment. The objectives of the study are to: (i) determine the effectiveness of Well London for improving healthy eating, physical activity and mental wellbeing in deprived inner-city communities; (ii) examine the effects in population subgroups linked to health inequalities in the UK.

Methods We used a pair-matched, cluster-randomised trial with 20 control neighbourhoods matched within London boroughs to 20 programme delivery neighbourhoods. The trial outcomes in adult residents (aged $\geq 16$ years) were collected using a structured electronic household survey, administered by fieldworkers to 100 randomly sampled residents in each intervention and control neighbourhood. The main outcome measures were: physical activity: meeting UK Chief Medical Officer-recommended five sessions of 30 minutes moderate intensity activity per week (selfreport International Physical Activity Questionnaire); healthy eating: eating at least five portions of fruit/vegetables per day (food frequency questionnaire from the Health Survey for England); and mental wellbeing: abnormal score on 12-item General Health Questionnaire; Warwick Edinburgh Mental Wellbeing Scale score.

Results The baseline survey in 2008 showed that the intervention and control populations are comparable on socio-demographic/economic characteristics and primary trial outcomes. At baseline, $37 \%$ of adults met the five-a-day (healthy eating), $60 \%$ met the five-aweek (physical activity), and 18\% reported experiencing anxiety or depression. Results from the follow-up survey will be available in April 2012. We will present the effects of Well London on the primary outcomes and subgroup analyses by gender, age, ethnicity and level of education.

Conclusion In a health system where less than $1 \%$ of the research budget is spent on primary preventive interventions for non-communicable diseases, robust evidence about the effectiveness and cost-effectiveness of upstream interventions is essential for action on health inequalities and reductions in healthcare spending recommended by the Marmot Review (2010) and the Wanless report (2004).

\section{OP07 WHAT SHAPES PARTICIPATION IN A COMMUNITY- BASED INTERVENTION? EVIDENCE FROM A QUALITATIVE EVALUATION OF THE WELL LONDON PROJECT}

doi:10.1136/jech-2012-201753.007

${ }^{1} \mathrm{~S}$ Jain, ${ }^{2} \mathrm{~A}$ Draper, ${ }^{3} \mathrm{~A}$ Clow, ${ }^{4} \mathrm{R}$ Lynch, ${ }^{2} \mathrm{~J}$ Derges. ${ }^{1}$ Social Work Subject Area, School of Social and Political Science, University of Edinburgh, Edinburgh, UK; ${ }^{2}$ Department of Human and Health Sciences, University of Westminster, London, UK; ${ }^{3}$ Department of Psychology, University of Westminster, London, UK; ${ }^{4}$ Department of Anthropology, UCL, London, UK

Background This paper examines how individual and area-level contextual factors shape participation in a community-based development and health promotion intervention. Well London was a 3 -year community development and health promotion programme for improving health behaviours (physical activity and healthy eating) and mental health and wellbeing in areas of high deprivation. The programme aimed to improve individual level health outcomes through a combination of neighbourhood and individual level interventions. Community engagement/participation was a central strategy of these interventions.

Methods A quantitative cluster randomised trial (CRT) was used to evaluate Well London in 20 neighbourhoods defined as Census Lower Super Output Areas (LSOAs). A qualitative study was nested within the trial to examine mechanisms and complexity. This study employed critical case sampling to select three intervention LSOAs that reflected a range of pre-existing community engagement and activities. In-depth semi-structured interviews were conducted with 59 respondents purposively sampled from each of 3 distinct areas. Each area reflected differences in implementation, nature of community life, and pre-existing community activities. Interviews addressed three topics: experiences of area, individual health \& wellbeing, and knowledge of and involvement in Well London. Transcripts were coded and thematic analysis undertaken using NVIVO software.

Results Analysis found that area level and individual-level characteristics interacted to shape specific models of individual participation in each area. In an area with a 'dispersed' community, limited pre-existing activities and implementation through formal institutions, participation was attributed by respondents to self-motivation and responses to deprivation. In contrast, in the 2 nd area, Well London implementation centred on an individual community organizer operating in a geographically close-knit area. Strong community interest and participation was shaped by the ability of this individual to inspire a sense of change. Finally, in an area with a 'saturation' of pre-existing activities, participation in Well London was part of a socially accepted pattern of community involvement. For new people to the area, involvement was viewed as aiding integration while for long-standing residents this was seen as a strategy to contribute to community life.

Conclusion Recent reviews on community participation present evidence of a causal link between participation and positive health outcomes. However, the mechanisms underlying this are not clear. The reasons people participate in Well London are shaped by interactions between individual and area-level factors. This suggests that understanding the link between community participation and health outcomes requires a contextualized analysis of why people participate and the meanings they associate with this.

\section{OP08 EVALUATING THE HEALTH INEQUALITIES IMPACT OF THE NEW DEAL FOR COMMUNITIES INITIATIVE}

doi:10.1136/jech-2012-201753.008

1J Popay, ${ }^{2} \mathrm{M}$ Whitehead, ${ }^{3} \mathrm{H}$ Badland, ${ }^{4} \mathrm{M}$ Stafford, ${ }^{5} \mathrm{~J}$ Nazroo, ${ }^{6} \mathrm{C}$ Dibbens, ${ }^{1} \mathrm{E}$ Halliday, ${ }^{2} \mathrm{~S}$ Povall. 'Division of Health Research, University of Lancaster, Lancaster, UK; ${ }^{2}$ Institute of Health, Psychology and Society, University of Liverpool, Liverpool, UK; ${ }^{3}$ Centre for Physical Activity and Nutrition, Auckland University of Technology, Auckland, Australia; 
${ }^{4}$ MRC Unit for Lifelong Health and Ageing, UCL, London, UK; ${ }^{5}$ School of Social Sciences, University of Manchester, Manchester, UK; ${ }^{6}$ Department of Geography and Sustainable Development, University of St. Andrews, St. Andrews, UK

Background New Deal for Communities (NDC), a 10-year areabased regeneration initiative begun in 1999 in 39 disadvantaged neighbourhoods in England, has the potential to reduce health inequalities because it focuses on key social determinants of these inequalities: unemployment, crime, education, housing and the physical environment. This study assesses whether the NDC initiative impacted on health inequalities across the socioeconomic spectrum of areas in England.

Methods The study primarily utilises secondary data including household surveys undertaken as part of a national evaluation of the NDC initiative led by Sheffield University. Representative samples of 500 residents per NDC area and matched comparator area in 2002, 2004, 2006 and 2008 provide data on health, lifestyles, wellbeing, demography and social determinants of health. The Health Survey for England in the same years provides information for representative samples drawn from across the socioeconomic spectrum, here categorised as highest, middle and lowest tertile of area deprivation. Logistic regression was used to estimate baseline differences in health and its social determinants, the time trend and differential time trends in NDC and non-intervention areas adjusted for sex, age, ethnicity and baseline education based on over 125,000 observations across eight years. Outcomes of interest included selfrated health, mental health inventory, smoking, employment status and housing tenure.

Results Initial analyses indicated a higher likelihood of smoking in non-intervention areas of medium deprivation (OR; 95\%CI: 1.33 ; $1.10,1.60)$, non-intervention areas of high deprivation $(1.80 ; 1.48$, $2.19)$, matched comparator areas $(1.77 ; 1.46,2.15)$ and NDC areas $(1.97 ; 1.68,2.31)$ compared with reference areas of low deprivation. Smoking declined over time but there was no evidence of differential rates of decline in the different areas. The likelihood of unemployment was considerably higher in all areas compared with the reference (for example, 4.29; 3.55, 5.18 in NDC areas). The likelihood of unemployment dropped over time in NDC areas but not in other areas (OR for time by NDC interaction: 0.93; 0.89, 0.96). Results for other outcomes are also available.

Conclusion The study indicates that the NDC intervention may have contributed to reducing a key social determinant of health inequalities, namely unemployment. Similar reductions were not observed in non-intervention high, medium or low deprivation areas in England which may eventually feed through into a narrowing of health inequalities between NCD and other areas. This initial work will be extended to examine impact across different intervention approaches and historical and current contexts.

\section{Population Based Studies: Early Life I}

\section{OP09 LIFE-COURSE BODY MASS INDEX, BIRTHWEIGHT AND LIPID LEVELS IN MID-ADULTHOOD: A NATIONWIDE BIRTH COHORT STUDY}

doi:10.1136/jech-2012-201753.009

SM Pinto Pereira, C Power. Centre for Paediatric Epidemiology \& Biostatistics, UCL Institute of Child Health, London, UK

Background Cardiovascular disease (CVD) is a major public health problem. Clinical trials have shown that lowering total- and/or lowdensity lipoprotein (LDL)-cholesterol reduces the risk of CVD. Therefore, to reduce the burden of CVD, improvement in lipid profiles is an important goal. To achieve this, a better understanding is needed of biological pathways leading to lipid levels. Lipid levels track from childhood to adulthood, implying that influences on adult cholesterol levels begin at young ages. There is scant evidence on the role of growth, including trajectories of body mass index (BMI) across the life-course with respect to lipids. We aimed to determine whether there are phases of the life-course that are particularly important for adult lipid levels.

Methods In the 1958 British Birth Cohort weight and height were recorded at birth (weight only), 7y, 11y, 16y, 23y, 33y and 45y. In 3,927 men and 3,897 women we assessed whether (i) birthweight was associated with $45 y$ lipids and whether associations were due to catch-up growth to 7y; (ii) there were particular periods when BMI, or change in BMI, influenced subsequent lipid levels; (iii) associations between current BMI and lipid levels were modified by BMI at younger ages; and (iv) duration of obesity was associated with lipid levels. Analyses were sex specific, adjusted for covariates and used multiple imputation to account for missing data.

Results Birthweight was inversely associated with triglycerides and in women with total- and LDL-cholesterol; associations persisted after adjustment for $7 y$ BMI. Associations with lipids strengthened with age of BMI measurement, e.g. per standard deviation (SD) higher BMI at 11y and 45y triglycerides were higher by $4.01 \%$ (95\% confidence interval: $2.26 \%, 5.75 \%)$ and $19.75 \%(18.18 \%$, $21.32 \%)$ respectively in women. BMI change was related to lipids, with strongest associations for the interval preceding lipid measurement: per SD increase in BMI 33-45y total-cholesterol and triglycerides were higher by $\sim 3 \%$ and $\sim 21 \%$ respectively. Associations between 45y BMI and lipids were stronger for those with lower than higher BMI at younger ages (P for interactions: $<0.01$ to $\leq 0.05$ ). This effect-modification was seen from childhood $7 \mathrm{y}$ in women, $11 y$ in men). Obesity in childhood but not thereafter and longer duration of obesity were unrelated to adult lipid levels.

Conclusion Our study suggests that life-course trajectories of body size influence adult lipid levels, with the consequences of a high adult BMI for adult lipid levels being particularly adverse for those with lower BMI at earlier life-stages.

\section{OP10 EFFECTS OF SOCIO ECONOMIC POSITION IN CHILDHOOD AND ADULTHOOD ON CARDIOMETABOLIC RISK FACTORS THE JERUSALEM PERINATAL FAMILY FOLLOW-UP STUDY}

doi:10.1136/jech-2012-201753.010

'O Manor, 'B Savitsky, 1,2H Hochner, ${ }^{3} V$ Meiner, ${ }^{2,4} \mathrm{DS}$ Siscovick, 'Y Friedlander. ${ }^{1} B r a u n$ School of Public Health, The Hebrew University-Hadassah Medical Center, Jerusalem, Israel; ${ }^{2}$ Department of Epidemiology, University of Washington, Seattle, Washington, USA: ${ }^{3}$ Department of Human Genetics, The Hebrew University-Hadassah Medical Center, Jerusalem, Israel; ${ }^{4}$ Department of Medicine, University of Washington, Seattle, Washington, USA

Background Studies indicate that Socio Economic Position (SEP) might be an important predictor of cardiometabolic disease mortality and morbidity; yet the importance of timing of the effect of SEP on cardiometabolic risk factors has not been fully established. Our aim was to assess the independent effect of SEP in both childhood and adulthood on cardiometabolic risk factors in young adults and to examine potential interactive effects of SEP at both time points on these risk factors.

Methods This is a prospective follow-up of 1132 individuals, born in Jerusalem between the years 1974-76, to young adulthood with bio-medical data at mean age 32. Outcomes include Body Mass Index (BMI), Waist to Hip Ratio (WHR), blood pressure, waist circumference (WC), pelvis circumference, body fat percentage, fasting levels of Triglyceride (TG), HDL-C, LDL-C, glucose, insulin, and lifestyle characteristics, including smoking and physical activity. SEP in childhood was based on father's occupation (scale 1-6) and in adulthood, on subject's occupation (scale 1-5). Additional analyses used maternal and subject's years of education. GLM and logistic regression models were used with mutual adjustment for SEP at both time points and further adjustment for gender, ethnic origin 